\title{
Jóvenes en Bachilleratos Populares: entre la individuación y la subjetivación política
}

\section{Young people in Popular High Schools: Between individuation and political subjectivation}

\author{
Shirly Said \\ CIS-CONICET/IDES \\ shirlysaid@gmail.com \\ Código Orcid: https://orcid.org/0000-0002-7937-8772
}

\begin{abstract}
Resumen
Este trabajo busca contribuir a la noción de subjetivación política a partir de los aportes de las sociologías del individuo para abordar las trayectorias educativas de jóvenes que estudian en Bachilleratos Populares. Se parte de la hipótesis de que existe una distancia entre las expectativas iniciales de los estudiantes jóvenes y la propuesta institucional de estas escuelas, que puede expresarse como una tensión latente entre procesos de individuación y de subjetivación. Se interroga cómo estudiar los procesos de individuación, en los que la prueba escolar resulta nodal, sin desatender los procesos de subjetivación política que se presentan como un horizonte posible pero no certero. En términos metodológicos, se indaga acerca de cómo abordar las experiencias de estos jóvenes desde una actitud de vigilancia epistemológica y se reflexiona acerca de las potencialidades del enfoque biográfico. Se concluye que esta perspectiva puede brindar herramientas teórico-metodológicas acordes a los objetivos de la investigación, priorizando la construcción de relatos e historias de vida.
\end{abstract}

\section{Palabras clave}

Juventud desfavorecida, individuos, política, Sociología de la educación, educación de adultos, metodología.

\begin{abstract}
In this article we seek to relate the concept of political subjectivation with the contributions of the sociologies of the individual, looking forward to generating theoretical and methodological tools that enrich a located analysis of young people's experiences in Popular High Schools in Argentina. Our main hypothesis is that there is a distance between the students' initial expectations and these schools' institutional proposal, that can be expressed as a latent tension between individuation and subjectivation processes. It questions how to study youngsters' individuation processes, in which the scholar trial is crucial, without disattending the political subjectivation processes presented as possible. In methodological terms, it inquires how to approach young people's experiences with an attitude of epistemological surveillance, and reflects on the potentialities of the biographic approach. It concludes that this perspectiwve can provide with suitable theoretic-methodological tools for the objectives of this research, giving priority to the production of life stories.
\end{abstract}

Keywords

Socially disadvantaged youth, individuals, politics, Educational sociology, adult'` education, methodology.

Forma sugerida de citar: Said, Shirly (2018). Jóvenes en Bachilleratos Populares: entre la individuación y la subjetivación política. Universitas, 28, pp. 141-157. 


\section{Introducción y estado de la cuestión}

Desde finales del siglo XX la educación ha cobrado una enorme centralidad en las luchas y en el debate público sobre las problemáticas sociales de América Latina, visibilizando la estrecha vinculación entre pedagogía y política. Frente a los efectos más críticos de la globalización sobre los proyectos estatales nacionales, las instituciones tradicionales y las identidades políticas e históricas, este (re)encuentro se ha expresado en las últimas décadas en movilizaciones estudiantiles y docentes en defensa del derecho a la educación, así como en la emergencia de nuevas iniciativas pedagógicas en el seno de movimientos y organizaciones sociales, experiencias educativas de fuerte impronta prefigurativa (Ouviña, 2011) que cuentan entre sus horizontes la formación de sujetos políticos.

En el caso de la Argentina, una de las experiencias más significativas y de mayor resonancia, regida por una recuperación de la impronta política y emancipatoria de la educación, es la de los Bachilleratos Populares, escuelas de nivel medio para jóvenes y adultos, que comenzaron a surgir en 2004 en organizaciones territoriales y fábricas recuperadas.

Tras el colapso social producido por la desarticulación del Estado en los años 90 , gran parte de las organizaciones sociales y políticas comenzaron a integrar sus luchas reivindicativas con la construcción cotidiana del mundo por-venir en términos de educación, trabajo, vivienda, salud, etc. Al iniciarse, en 2003, un período de mayor intervención estatal que implicó el despliegue de múltiples estrategias del Estado "dentro de los propios territorios donde estos movimientos sociales se habían consolidado en los años del "ciclo de protesta' entre 1993-2003” (Aguiló y Wahren, 2014, p. 99), muchas de estas organizaciones apostaron a ampliar su despliegue territorial.

Como parte de aquel proceso, y en el marco del surgimiento de iniciativas educativas impulsadas en y por movimientos sociales en todo el continente ${ }^{1}$, en la Argentina los Bachilleratos Populares de Jóvenes y Adultos (en adelante, BPJA) se plantearon un horizonte dual: la construcción de poder popular desde los territorios, mediante la praxis de la educación popular

1 Entre las más significativas se encuentran las escuelas del Movimiento Sem Terra (MST) de Brasil, el Sistema Educativo Rebelde Autónomo Zapatista del Ejército Zapatista de Liberación Nacional (EZLN) en México, la Universidad Intercultural de las Nacionalidades y Pueblos Indígenas "Amawtay Wasi", impusalda por la CONAIE en Ecuador y la Universidad Campesina del Movimiento Campesino de Santiago del Estero (MOCASE-VC). 
y la organización autogestiva, y -en simultáneo- la interpelación al Estado como garante del derecho de todos los sectores sociales a la educación (Elisalde, 2008), motivo por el que reclaman el reconocimiento estatal.

En la actualidad existen más de 96 BPJA en todo el país ${ }^{2}$, que se definen a sí mismos como autogestionados, con autonomía política y pedagógica respecto del Estado, y llevan adelante un proyecto político-pedagógico ligado a la educación popular latinoamericana de impronta emancipatoria, tendiente a promover formas de ciudadanía activa y política entre los estudiantes, y la formación de personas conscientes de la realidad en la que viven (Rubinsztain, 2012) y capaces de constituirse como sujetos políticos con plenos derechos (Elisalde, 2008).

En este contexto, el campo de los estudios sobre BPJA se ha ampliado visiblemente en los últimos años -en el marco de la expansión del área de conocimiento sobre educación "en y desde los movimientos sociales" (Michi, Di Matteo y Vila, 2012)- y es posible identificar una rica variedad de enfoques y temas de interés. Sin embargo, consideramos que la cuestión de la subjetivación política no ha sido suficientemente abordada y problematizada desde la experiencia de los estudiantes: es frecuente encontrar estudios que aluden al tipo de sujetos que los BPJA se proponen formar, definidos como "sujetos políticos", "críticos", "conscientes", "participativos", "reflexivos", etc. (Elisalde, 2008; Areal y Terzibachian, 2012; Rubinsztain, 2012), pero pocos dan cuenta de la relación entre esos objetivos y los deseos, expectativas y experiencias de los educandos.

A su vez, la producción académica que aborda la subjetivación política en clave juvenil en estas escuelas es relativamente escasa. Ante la juvenilización de la población de la Educación de Jóvenes y Adultos en general, y la simultánea visibilización de la juventud como actor social y político (Kriger, 2014), creemos necesario focalizar el estudio en esta población ${ }^{3}$. Al respecto, recuperamos los estudios que plantean que el modelo institucional y pedagógico de los BPJA repercute de manera particular en las trayectorias

2 El Primer Relevamiento Nacional de Bachilleratos Populares, realizado por el GEMSEP (Grupo de Estudios sobre Movimientos Sociales y Educación Popular) en 2015, da cuenta de la existencia de 93 BPJA. Desde ese momento, al menos tres nuevas escuelas han abierto con esta modalidad, en la provincia de Río Negro y en la Ciudad de Buenos Aires.

3 Desde el surgimiento de los BPJA se reconoce que la población estudiantil es más joven que adulta (Elisalde, 2008), en sintonía con la situación de la Educación de Jóvenes y Adultos (EDJA) durante las últimas dos décadas (Sirvent, 2007). 
educativas de los jóvenes (Mereñuk, 2011), y que los predispone "positivamente a la participación, generando actitudes portadoras de 'potencialidad política"” (Kriger y Rybak Di Segni, 2011, p. 6) que propician la concientización de su condición de "sujetos jóvenes y nuevos ciudadanos" (p. 6).

Buscamos complejizar estos trabajos desde una perspectiva que pueda abordar las experiencias juveniles sin pretender que sean, de antemano, liberadoras, emancipadoras o transformadoras de la subjetividad.

El presente artículo busca vincular, entonces, la noción de subjetivación política con los aportes de las sociologías del individuo, para nutrir análisis situado de las experiencias de jóvenes en Bachilleratos Populares. En el marco de una investigación doctoral ${ }^{4}$ en curso, nos proponemos acuñar nuevas herramientas conceptuales y compartir las reflexiones que guiaron la elección del enfoque biográfico como perspectiva teórico-metodológica. En la actualidad nos encontramos realizando el trabajo de campo, por lo que no trabajaremos aun sobre hallazgos empíricos, sino sobre el armazón conceptual que sostiene nuestro trabajo.

Como plantea Larrondo (2012), retomando a Lahire, "la contradicción, la ambigüedad, la discordancia o las concordancias entre los jóvenes y las escuelas no resultan un fenómeno extraño: es parte de la lógica del vínculo entre socialización, percepción y acción de los sujetos en cualquier tipo de institución" (2012, p. 21). Para el caso de los Bachilleratos Populares, García (2011) señala que en las investigaciones se suelen homogeneizar las posiciones de docentes y estudiantes, y Gluz (2013) observa que "tanto la militancia como la formación de sujetos políticos tiene una fuerza en el discurso docente que no encuentra correlato en los estudiantes" (2013, p. 70).

Planteamos entonces que para abordar las experiencias de formación de jóvenes en Bachilleratos Populares es necesario hacer visible la distancia entre sus expectativas iniciales -en gran medida ligadas a demandas pragmáticas como titulación, inclusión laboral y movilidad social (Brusilovsky, 2005) - y la propuesta institucional. Es por ello que consideramos necesario reflexionar acerca de las potencialidades y limitaciones de la noción de subjetivación política para abordar las experiencias escolares de estos jóvenes,

4 Se trata del proyecto de doctorado "Movimientos sociales y educación: un estudio diacrónico sobre propuestas y experiencias de subjetivación política juvenil en Bachilleratos Populares”, dirigido por la Dra. Miriam Kriger. 
y allí las sociologías del individuo (Martuccelli y De Singly, 2012) habilitan un vasto terreno de indagación.

Como punto de partida, esta perspectiva teórica parte de identificar un "creciente proceso de desincrustación social de los patrones biográficos, asociado a los procesos de individualización" (Saraví, 2009, p. 29) propios del debilitamiento de las instituciones sociales en el período post-industrial. En función de ello, plantea que la base problemática de la experiencia individual en la cultura contemporánea es que se le exige a los individuos el manejo autónomo de sus experiencias biográficas, con niveles inéditos de reflexividad y sensibilidad (Saraví, 2015).

Dentro de esta línea, recuperaremos las nociones de individuación y subjetivación que Danilo Martuccelli (2007) identifica como dos "vías del individuo sociológico" (2007, p. 19). La primera estudia el proceso mediante el cual la sociedad se dota de individuos, que -aunque con múltiples variantes- es atravesado por todos los seres humanos. A diferencia de ella, la segunda se centra en la figura moral de la emancipación como horizonte, como una conquista posible pero no certera que, por lo tanto, no abarca a todos los individuos (Martuccelli, 2010).

Nos preguntamos por las implicancias de que la subjetivación aparezca como un horizonte explícito, materializado en propuestas concretas en los BPJA, con ciertas pautas específicas acerca del tipo de sujetos -políticos, críticos, conscientes- que se busca formar.

En este sentido, y partiendo de la hipótesis de que la tensión entre los procesos de individuación y de subjetivación resulta constitutiva de la experiencia de los y las jóvenes que estudian en BPJA, buscamos acuñar herramientas teórico-metodológicas que permitan dar cuenta de esta coexistencia, recuperando elementos de ambos enfoques analíticos. Primeramente, nos cuestionamos: ¿Cómo analizar los procesos de individuación de los jóvenes, considerando sus experiencias específicas frente a la prueba de la escolaridad, pero sin desatender el estudio de los procesos de subjetivación que se les presentan como un horizonte posible? O, a la inversa, ¿cómo estudiar los procesos de subjetivación que pueden atravesar los jóvenes, sin caer en una generalización que obture las singularidades de sus "procesos de fabricación" (Martuccelli, 2010, p. 9) como individuos? En segundo lugar, en términos metodológicos, surge la pregunta por cómo abordar las experiencias de los jóvenes desde una actitud de vigilancia epistemológica (Bachelard, 1979) que evite el sesgo que ha caracterizado a los procesos formativos 
en los BPJA como productores de sujetos políticos, generalmente producido por una identificación con el objeto de estudio.

Consideramos que estos interrogantes contribuyen a la construcción de herramientas conceptuales referenciadas en la sociología de la educación que pueden enriquecer el campo de las pedagogías críticas -más allá del caso específico de los BPJA y del contexto socio-educativo de la Argentinay permiten abordar bajo una nueva óptica el estudio de experiencias escolares de Educación Popular, formación ciudadana y/o formación política de jóvenes de sectores desfavorecidos.

\section{El desafío de la individuación: exigencias y experiencias en singular}

Desde finales de siglo XX a la actualidad, la era post-industrial ha sido caracterizada por diversos autores europeos como modernización reflexiva (Beck, 1997), "alta modernidad" (Giddens, 1997, p. 118) y, entre otros, “desmodernización”, término acuñado por Touraine (1997, p. 27) que propone la existencia de dos procesos simultáneos, la desinstitucionalización y la des-socialización, que implican la pérdida de recursos identitarios y, fundamentalmente, un clima de riesgo e incertidumbre para los actores sociales. En otras palabras, se plantea que el debilitamiento de las instituciones y de los factores estructurales que en épocas previas podían contener a los sujetos ha llevado a un desenmarcamiento de los individuos que, contra las pretensiones del funcionalismo, ya no encuentran un rol predefinido a desempeñar en la sociedad ${ }^{5}$

En relación con nuestro problema, resulta interesante la propuesta de estudiar las pruebas comunes que atraviesa una sociedad y que se plantean como desafíos que "circunscriben un conjunto de grandes retos estructurales, particularmente significativos" (Araujo y Martuccelli, 2010,p. 84). Esas pruebas son propias de cada sociedad, asociadas -aún a pesar de la globa-

5 La situación latinoamericana presenta particularidades que no pueden homologarse exactamente al proceso europeo, dado el alcance diferencial que ha tenido los estados de bienestar en ambos continentes y, por ende, el desigual impacto de su debilitamiento. No ahondaremos aquí en la especificidad de los procesos de individuación en América Latina, pero recuperaremos trabajos que dan cuenta de ellos, poniendo el foco en los jóvenes en contextos de desigualdad social (Saraví, 2009 y 2015; Di Leo y Camarotti, 2013) 
lización- a fronteras nacionales (Martuccelli y De Singly, 2012), de modo tal que el trabajo que los individuos realizan para afrontarlas se constituye como el principal vector de su proceso de individuación. En este sentido, la experiencia del éxito o el fracaso en la superación de esas pruebas (con la infinidad de vicisitudes que pueden conllevar) marca las trayectorias de los individuos y, en función de las respuestas y "los recursos que movilizan en un contexto específico de determinaciones sociales" (Araujo y Martuccelli, 2010, p. 87), les da forma.

Según esta perspectiva, la primera prueba social y existencial que enfrenta el individuo moderno es "lograr sostenerse en un mundo que no lo contiene más con la fuerza con que lo hizo en otros períodos" (Martuccelli, 2007, p. 66), en un contexto en el que prima el mandato social de ser autónomo e independiente, es decir; dueño de sí mismo y capaz de auto-sostenerse. Citando a Beck, Martuccelli (2007) plantea al respecto que "las principales instituciones de la sociedad (el trabajo, el empleo, la escuela, la familia) estarían cada vez más orientadas hacia el individuo, obligando a cada persona a desarrollar y asumir su propia trayectoria biográfica" (2007, p. 33). En este contexto, dado que la creencia en el ideal del individuo soberano está tan arraigada, los soportes - vínculos, actividades, espacios y elementos materiales e inmateriales que sostienen al individuo en el mundo- son invisibilizados para demostrar solidez y autosuficiencia.

\section{La subjetivación como horizonte: en busca del potencial de emancipación}

Partiendo de una concepción de la subjetivación política ligada a una construcción de tipo cultural y no evolutiva, según la cual los hombres y mujeres son siempre sujetos sociales pero no necesariamente llegan a ser sujetos políticos plenos (Kriger, 2014), en un trabajo previo (Said y Kriger, 2014) hemos puesto en diálogo a Freire y Rancière. Desde enfoques y campos disciplinares distintos, ambos dan cuenta de las tensiones sujeción/ emancipación, individual/colectivo en el proceso de subjetivación política, y coinciden en identificar que la subjetivación política consiste en la verificación de la igualdad de los seres humanos; y que se conquista en un acto comunicativo.

En cuanto al primer punto, tomamos de Rancière el término efecto de igualdad y lo encontramos equiparable a lo que Freire define como huma- 
nización, en tanto ambos se refieren a la forma en que los oprimidos, los incontados, se constituyen como sujetos en cuanto logran hacerse presentes, para los otros y para ellos mismos, como seres con iguales capacidades y condición de humanidad que aquellos que hasta ese momento los negaban. Ese proceso no es otra cosa que la subjetivación, la afirmación del "nos sumus, nos existimus" cartesiano en plural (Rancière, 2010, p. 52), en el que el campo de la experiencia se reconfigura por completo, en términos colectivos e individuales. Es a partir de esto que Rancière (2010) asocia el efecto de igualdad a la emancipación (2010, p. 51), así como Freire (2009) vincula la humanización con la "búsqueda del ser más" (2009, p. 41), y a ésta con lucha por la liberación.

La segunda coincidencia radica en el espacio simbólico en el que sucede la subjetivación: ambos plantean que el encuentro no solo está mediado por la palabra sino que ocurre en ella. Esto no significa, sin embargo, que se hable de un terreno abstracto y desvinculado de la práctica, sino lo contrario. Para Freire, los hombres ganan significación como tales en el diálogo y, desde su perspectiva, diálogo y palabra son una sola cosa: "la unión inquebrantable entre acción y reflexión", es decir, la praxis que transforma al mundo (Freire, 2009, p. 97). En el planteo de Rancière, la palabra introduce la distorsión de la lógica policial en la lógica política, un acontecimiento verbal que lleva a los individuos a subjetivarse cuando prueban "la distancia entre su condición de animales dotados de voz y el encuentro violento de la igualdad del logos" (Rancière, 2010, p. 54).

Creemos central recuperar esta trasformación del individuo en sujeto para profundizar la propuesta de la sociología de la individuación. Tal como plantea Martuccelli (2010), "el sujeto nunca precede, no es algo que está dado (...). Supone una lógica de emancipación explícita o implícita, es decir, no todos somos sujetos, solo algunos logran serlo" (2010, p. 7). En este sentido, ser (hacer-nos) sujetos implica una proyección, posible pero no certera, que requiere un trabajo específico de los individuos, puestos a prueba por sus sociedades. Aquí la relación dialéctica entre reflexión y acción ocupa un papel fundamental para el proceso de subjetivación, que sin dejar de ser un reto colectivo, se convierte en una exigencia inmediata (aquí y ahora) para cada uno.

Esta conceptualización responde al propósito del autor de singularizar los análisis sin abandonar la visión de lo colectivo (Martuccelli, 2007), y atendiendo su vez a las múltiples tensiones ya enunciadas. Al respecto, concluye que en la matriz de la subjetivación "la relación consigo mismo es siempre 
estudiada como el resultado de una oposición entre las lógicas del poder y su cuestionamiento social" (2007, p. 29), es decir, como una puerta abierta a la emancipación y también franqueada por múltiples condiciones limitantes, sobre las que el individuo deberá trabajar -y superarlas- para hacerse sujeto.

\section{Entre individuación y subjetivación: la tensión ante la prueba escolar}

Como hemos visto, en las sociedades contemporáneas los individuos se enfrentan, ya sea concreta o potencialmente, a procesos de individuación y de subjetivación. Recuperando nuestra hipótesis, consideramos que los BPJA son un escenario privilegiado para la expresión de la tensión entre ambos procesos, que hace carne en los cuerpos de los estudiantes jóvenes.

Teniendo en cuenta que la obligatoriedad social y simbólica de la escuela secundaria en la Argentina se reafirma jurídicamente en 2006, con la sanción de la Ley 26.206, la finalización del nivel se ha consolidado como una exigencia ineludible para jóvenes y adultos. Esto constituye, en términos de Martuccelli, a la "prueba escolar" (Martuccelli, 2007; 2013), entendida como el desafío común en las sociedades contemporáneas de completar la educación obligatoria, que adopta características específicas en función de cada contexto social. Como plantea Nobile (2016), al incorporarse la secundaria obligatoria al patrón normativo que delimita la trayectoria vital de quienes están en edad de asistir a ella, "pasa a ser una exigencia para los jóvenes contemporáneos; es decir, una prueba estructural que están obligados a afrontar" (2016, p. 112).

Ahora bien, si la educación secundaria se ha convertido en una meta social en el contexto latinoamericano (Rivero, 2012), para los estudiantes de la modalidad de Educación de Jóvenes y Adultos (EDJA) ella representa un desafío aún mayor, dado que en gran medida los ha definido por la negativa: es frecuente encontrar relatos que califican la trayectoria escolar de estos jóvenes en términos de fracaso (Martuccelli y De Singly, 2012) por lo que no han podido hacer: sostener una escolaridad tradicional, idea que se apoya en trayectorias teóricas previamente estandarizadas que se corresponden cada vez menos con las trayectorias educativas reales de los adolescentes y jóvenes (Terigi, 2010). Esta evaluación negativa, rayana con la calificación moral y el estigma normalizante, suele ser promovida por el discurso públi- 
co, e incluso por el académico (Téllez Velasco, 2011) que alude a los jóvenes $n i$-ni-que no estudian

ni trabajan-. Lejos de reconocerlos como una población en situación de riesgo educativo (Sirvent, 2007) o de identificar "la falta de ofertas instituciones que entiendan la realidad que ellos viven cotidianamente" (Ramírez, 2013, p. 77), se cae en la responsabilización individual de estos jóvenes.

Ante esta "doble obligatoriedad” (D’Aloisio, 2014) -legal y simbólicade la escuela secundaria, y la vigencia de políticas públicas orientadas a garantizar su cumplimiento, la llegada de los y las jóvenes adolescentes a los Bachilleratos Populares está asociada al objetivo explícito de finalizar la secundaria y superar la prueba escolar, y suele responder a la recomendación de un familiar o amigo, priorizando la cercanía del hogar o lugar de trabajo y la posibilidad de compatibilizar la cursada con los horarios familiares y laborales, asistir con hijos/as a clase, entre otras, pero no a la especificidad de la propuesta político pedagógica. Simultáneamente, los jóvenes encuentran con una escuela enmarcada en una organización social, cuyo objetivo manifiesto es formar sujetos políticos a partir de prácticas colectivas de participación, decisión y organización, que difieren en múltiples aspectos de la imagen tradicional sobre la escuela secundaria y de las prescripciones sobre modos de ser estudiante.

Consideramos que este encuentro entre las necesidades de los estudiantes y los propósitos institucionales de los BPJA representa la tensión central de nuestro estudio: tomando de Araujo y Martuccelli (2010) la afirmación de que "el trabajo de los individuos para producirse como sujetos está condicionado por lo que le toca a la acción simultánea del ideal y de la experiencia social" (2010, p. 88), planteamos que si bien los objetivos de los BPJA pueden no responder, en primera instancia, a las expectativas personales de los jóvenes, su aparición contingente en la experiencia escolar, y el entrecruzamiento de estos ideales con los suyos propios -enmarcados en un diálogo más profundo entre los múltiples actores de la comunidad educativa (docentes, militantes, estudiantes, familias, etc.) - influyen de forma singular en su trabajo para producirse como sujetos y en la construcción de su identidad personal y social (Kriger y Fernández Cid, 2011). Esto coincide con la comprensión de la politización como un proceso psicosocial complejo, de carácter individual y colectivo, intra e intersubjetivo, en permanente movimiento (Kriger, 2014), cuyo análisis exige un abordaje multidimensional y situado.

Dado que el foco está puesto en las experiencias de los propios jóvenes al atravesar estos procesos, surge entonces la pregunta por cómo abordar- 
las, conocerlas, llegar a ellas. ¿Cómo acceder a la particular constelación de elementos que compone cada proceso de constitución de los jóvenes como individuos y, quizá también -aunque no necesariamente- como sujetos políticos? ¿Cómo saber si la participación, la organización, la política, tan centrales en el discurso institucional de los Bachilleratos Populares, y en particular en nuestro estudio, resultan significativas para los estudiantes que las vivencian y, por tanto, constitutivas de su experiencia?

\section{El enfoque biográfico como propuesta de abordaje}

Dubet y Martuccelli (1998) plantean que en la escuela los actores se socializan a través de diversos aprendizajes, como la integración de la cultura escolar, la construcción de estrategias sobre el mercado escolar, el manejo subjetivo de los conocimientos y las culturas que los portan] y se constituyen como sujetos en su capacidad de manejar su experiencia. En este sentido, señalan, "toda educación es (...) un trabajo sobre sí mismo" (1998, p. 14). Este trabajo es definido como una "experiencia social" (p. 15) que involucra la voluntad del actor para definir su situación y construir imágenes de sí mismo. Se trata de poner en juego un "distanciamiento de sí, una capacidad crítica y un esfuerzo de subjetivación" (p. 15).

De ello se derivan dos conclusiones simples pero fundamentales: a) que existe una multiplicidad de aspectos de la vida escolar que exceden ampliamente la propuesta formalizada en el currículum; y b) que esa variedad de vivencias exige de los individuos una considerable capacidad de agencia para atravesarlas, darles sentido e integrarlas. Tal tarea inherentemente reflexiva y crítica, es llamada experiencia, y constituye la principal herramienta de construcción de la subjetividad.

El aporte que deseamos realizar radica especialmente en abordar aquello que escapa, trasciende, y/o cuestiona los objetivos manifiestos de ciertas propuestas ligadas a la educación popular. En este sentido, si bien coincidimos con Langer y Levy (2009) en que los BPJA, al ubicar "la participación como contenido principal a enseñar y a aprender, vuelven el 'currículum oculto' no tan oculto" (2009, p. 245),

consideramos que es necesario focalizar en aquello que, más allá de los esfuerzos de organizadores, docentes e impulsores de estas escuelas por pro- 
mover la des-ocultación, ocurre pero no es dicho (o, incluso, no es decible), en relación a la participación y a la política.

Esto refuerza la necesidad de documentar los aspectos no documentados (Rockwell, 2009) de la experiencia escolar a través de las voces de sus protagonistas. En continuidad con lo planteado por Freire y Rancière respecto del lenguaje como lugar para el proceso de subjetivación, consideramos que el enfoque biográfico (Arfuch, 2002) pueden posibilitar un abordaje muy interesante, asentado en el potencial subjetivante de la propia narración de sí -y del "nosotros"-.

Para comenzar, el concepto de biografización de Delory Momberger (2014) reconoce que la narración es previa a la enunciación y que consiste en una actividad constante de construcción de experiencia a partir de las situaciones vividas. A partir de ello, la narración "(auto)biográfica”, es "una modalidad privilegiada de las operaciones de biografización” (2014, p. 699), que involucra profundos procesos de reflexividad. En la misma senda, Scott (2001) propone "rehusarse a una separación entre la experiencia y el lenguaje, y en su lugar insistir en la cualidad productiva del discurso" (2001, p. 65). Con ello, afirma que los sujetos "tienen agencia" (p. 66) y se constituyen discursivamente.

En el estudio de experiencias educativas, Suárez (2014), plantea que la "expansión de las prácticas narrativas (auto)biográficas ha contribuido a la emergencia de nuevos sujetos, experiencias y discursos pedagógicos que disputan sentidos y legitimidad respecto de cómo nombrar, hacer y pensar la educación y la formación" (2014, p. 764). Al presentar a los docentes como narradores de sus propias experiencias escolares, el autor señala que:

Dejan de ser los que eran, se trans-forman, son otros. Asumen una posición reflexiva que desafía las propias comprensiones, reconfigura las propias trayectorias profesionales y resignifica las propias acciones e interpretaciones sobre la escuela (...) Narrar es pensar y re-pensar por escrito sobre nuestras prácticas, nuestras vidas, nuestros mundos, es re-inventarlos al volverlos a nombrar pero con otras palabras, es formar-se junto con otros (Suárez, 2011, p. 19).

Desde nuestra perspectiva, el proceso que describe, al estar asociado a lo que ocurre en la narración, bien puede extrapolarse al relato autobiográfico sobre la experiencia escolar juvenil.

En el campo de la sociología, Di Leo y Camarotti (2013) recuperan la categoría de pruebas propuesta por Martuccelli y proponen abordar las vidas de 
los jóvenes desde una perspectiva que contemple "la diversidad de situaciones y las interconexiones complejas y hasta contradictorias en que se constituyen sus identidades individuales y sus experiencias sociales" (2013, p. 21). Es así que recuperan la propuesta metodológica de Leclerc-Olive (2009) para la construcción de relatos autobiográficos. Ante la propuesta inicial de escribir y publicar su historia de vida, los investigadores llevan adelante entrevistas sucesivas con los jóvenes, en las que comienzan con la pregunta acerca de quiénes son y cómo se describirían -su "autoidentificación subjetiva" (Di Leo y Camarotti, 2013, p. 23), y pasan luego a reconstruir los giros de la existencia -momentos o situaciones que marcaron sus vidas- considerando como significativos aquellos acontecimientos concebidos como tales "por los propios entrevistados y no por el investigador" (2013, p. 24).

En resumen, los enfoques presentados comparten el objetivo de acercarse a los procesos de construcción de las identidades y las experiencias sociales de los sujetos mediante procesos de reflexividad que priorizan la voz de los protagonistas, y en los que el trabajo es siempre activo e inacabado. A los fines de nuestra investigación, consideramos que resultan de una riqueza invaluable para mantener una actitud de vigilancia epistemológica (Bachelard, 1979) en el análisis de las experiencias de jóvenes que estudian en Bachilleratos Populares, habilitando simultáneamente el registro de los sentidos asignados a la superación de la prueba escolar y a los procesos de transformación individual y colectiva que pueden implicar los procesos de subjetivación política.

\section{Consideraciones finales y perspectivas}

A lo largo de este trabajo hemos considerado el aporte que pueden hacer los conceptos de individuación y subjetivación de Martuccelli a los estudios sobre espacios de educación popular. De alguna manera, la pregunta que nos guía es: ¿Cómo estudiar la subjetivación política en tiempos de individuación? ¿Cómo dar cuenta de la imbricación simultánea de estos procesos sin suponerlos, a priori, mutuamente excluyentes? ¿Cómo, en términos metodológicos, superar el sesgo de las prenociones sobre la educación popular y hacer lugar a aquello que se presenta como emergente en la experiencia de los jóvenes en Bachilleratos Populares?

Ante el primer interrogante, acerca de cómo estudiar los procesos de subjetivación política que pueden atravesar los jóvenes sin negar los desa- 
fíos propios de su construcción como individuos, hemos identificado ciertas bases para un posible encuentro: el eje estaría en analizar la forma en que los jóvenes que estudian en BPJA se individúan, a partir de experiencias múltiples y pruebas que trascienden el ámbito escolar, pero prestando una especial atención a la forma en que se apropian, rechazan y/o transforman para sí los ideales de subjetivación política (en clave emancipatoria y colectiva) propuestos por la institución. Esto permitiría conocer la forma en que aquellos ideales se inscriben en su trabajo como individuos, ya sea como experiencia social o como ideales internalizados.

En definitiva, es sobre esta construcción como sujetos que nos encontramos indagando empíricamente en el trabajo de campo, para acceder a los sentidos subjetivos que los jóvenes asignan a la experiencia educativa en el BPJA, poniendo el eje en el proceso de conformación de identidades individuales y colectivas. Es por eso que consideramos fundamental incorporar la perspectiva de los propios actores, escuchando las voces de los jóvenes y reconociendo sus resistencias.

Por otra parte, ante nuestra segunda pregunta acerca de cómo acceder a los significados subjetivos que los jóvenes asignan a sus experiencias en los BPJA, consideramos que el enfoque biográfico puede brindar herramientas teórico-metodológicas aptas para profundizar en nuestros objetivos de investigación, mediante la construcción de relatos e historias de vida. Dado que la experiencia no es "lo que pasa, o lo que acontece, o lo que llega, sino los que nos pasa, o nos acontece, o nos llega" (Larrosa, 2003, p. 168), nuestro interés radica en conocer la forma en que los jóvenes interpretan, se apropian, resisten y/o rechazan la propuesta institucional con marcos de sentido propios.

\section{Bibliografía}

Aguiló, V., \& Wahren, J. (2014). Los bachilleratos populares de Argentina como “campos de experimentación social". Argumentos, 27(74), 97-114.

Araujo, K. y Martuccelli, D. (2010). La individuación y el trabajo de los individuos. Educaçao e Pesquisa, 36, 77-91.

Areal, S. \& Terzibachian, M. F. (2012). La experiencia de los bachilleratos populares en la Argentina: exigiendo educación, redefiniendo lo público. Revista mexicana de investigación educativa, 17(53), 513-532.

Arfuch, L. (2002). El espacio biográfico: dilemas de la subjetividad contemporánea. Buenos Aires: Fondo de Cultura Económica. 
Bachelard, G. (1979). La formación del espíritu científico. México: Siglo XXI.

Beck, U. et al. (1997) Modernización reflexiva. Madrid: Alianza Universidad.

Brusilovsky, S. (2005) Educación de jóvenes y adultos (EDJA). Plan Fénix, FCE, UBA.

D’Aloisio, F. (2014). Mirar el porvenir a través de la secundaria: la concreción de proyectos biográficos, ¿Una cuestión de voluntad individual? En: H. Paulin, M. Tomasini, Jóvenes y escuela. Relatos sobre una relación compleja (pp.19-50). Córdoba: Editorial Brujas.

Delory-Momberger, C. (2014). Experiencia y formación: biografización, biograficidad y heterobiografía. Revista Mexicana de Investigación Educativa, 19(62), 695-710.

Di Leo, P. F. \& Camarotti, A. C. (2013). Quiero escribir mi historia: vidas de jóvenes en barrios populares. Buenos Aires: Biblos.

Dubet, F. \& Martuccelli, D. (1998). En la escuela. Sociología de la experiencia escolar. Buenos Aires: Losada.

Elisalde, R. (2008). Bachilleratos Populares en empresas recuperadas y organizaciones sociales. Revista Novedades Educativas, 209. Buenos Aires: Noveduc.

Freire, P. (2009). Pedagogía del oprimido. Buenos Aires: Ed. Siglo XXI.

García, J. (2011). Bachilleratos populares y 'autonomía': ¿espacios de la transformación o de la reproducción?. Boletín de Antropología y Educación, (2).

Giddens, A. (1997). Vivir en una sociedad postradicional. En: Modernización reflexiva. Madrid: Alianza Universidad.

Gluz, N. (2013). Las luchas populares por el derecho a la educación: experiencias educativas de movimientos sociales. 1a ed. Buenos Aires: CLACSO.

Kriger, M. (2014). Politización juvenil en las naciones contemporáneas. El caso argentino. Revista Latinoamericana de Ciencias Sociales, Niñez y Juventud, 12(2), 583-596.

Kriger, M., y Rybak Di Segni, N. (2011). "El espacio escolar como generador de subjetividades políticas. Resultados preliminares de un estudio empírico sobre el Bachillerato Popular de Jóvenes y Adultos IMPA Buenos Aires”. Primer Congreso de Comunicación/Ciencias Sociales desde América Latina: Tensiones y Disputas en la Producción de Conocimiento para la Transformación (COMCIS), FPCS-UNLP, La Plata.

Kriger, M. y Fernández Cid, H. (2011). "Los jóvenes y la construcción del 'ciudadano ideal”. III Congreso Internacional de Investigación de la Facultad de Psicología. La Plata. 
Langer, E. y Levy, E. (2009). Educación de jóvenes y adultos, política social gubernamentalidades en el siglo XXI. Revista de la Escuela de Ciencias de la Educación, 4, 229-250. UNR.

Larrondo, M. (2012). ¿Nuevos alumnos? La construcción de la identidad escolar en nuevos dispositivos de educación media. Un estudio de casos en contextos de pobreza de la Ciudad de Buenos Aires. Pensamiento Educativo. Revista de Investigación Educacional Latinoamericana, 49(1), 18-31.

Larrosa, J. (2003). “La experiencia y sus lenguajes”. Conferencia dictada en la Serie Encuentros y Seminarios, Barcelona, Departamento de Teoría e Historia de la Educación, Universidad de Barcelona.

Leclerc-Olive, M. (2009). Temporalidades de la experiencia: las biografías y sus acontecimientos. Iberofórum. Revista de Ciencias Sociales de la Universidad Iberoamericana, 8(6), 1-39.

Martuccelli, D. (2007). Cambio de rumbo. La sociedad a escala del individuo. Santiago de Chile: LOM.

Martuccelli. D. (2010). Entrevista: La sociología en los tiempos del individuo. Doble Vínculo, 1(1).

Martuccelli, D. \& De Singly, F. (2012). Las sociologías del individuo. Santiago de Chile: LOM.

Mereñuk, A. (2011). El lugar de las decisiones en las Trayectorias Educativas de Jóvenes próximos a egresar de los bachilleratos populares. En: C. Jacinto (Comp.), La construcción social de las trayectorias laborales de jóvenes. Políticas, instituciones, dispositivos y subjetividades. Buenos Aires: Ed. Teseo.

Michi, N., Di Matteo, J. \& Vila, D. (2012). Movimientos populares y procesos formativos. Polifonías Revista de Educación, 1(1), 22-41.

Nobile, M. (2016). La escuela secundaria obligatoria en Argentina: Desafíos pendientes para la integración de todos los jóvenes. Última década, 24(44), 109-131.

Ouviña,H. (2011). Especificidades y desafíos de la autonomía urbana desde una perspectiva prefigurativa. En: AA. VV, Pensar las autonomías. México: Sísifo.

Ramírez, R. (2013). El barrio, la escuela y la iglesia. En: P.F Di Leo y A.C. Camarotti, Quiero escribir mi historia. Vidas de jóvenes de barrios populares, Buenos Aires, Biblos.

Rancière, J. (2010). El desacuerdo. Política y filosofía, Bs. As., Ediciones Nueva Visión. 
Rockwell, E. (2009). La experiencia etnográfica. Historia y cultura en los procesos educativos. Buenos Aires: Paidós.

Rubinsztain, P. (2012). Los vínculos de la praxis freireana con una cultura política emergente en Argentina de 2001. Polifonías Revista de Educación, 1(1), 95-111.

Said, S. y Kriger, M. (2014). Subjetivación política y educación popular: La noción de diálogo en Rancière y Freire como aporte a la reflexión teóricometodológica sobre Bachilleratos Populares. Questión, 1(42), 405-420.

Saraví, G. (2009). Transiciones vulnerables. Juventud, desigualdad y exclusión en México. México: CIESAS.

Saraví, G. 2015. Juventudes fragmentadas. Socialización, clase y cultura en la construcción de la desigualdad. México: Flacso, CIESAS.

Scott, J. W. (2001). Experiencia. Revista de estudios de género: La ventana, 2(13), 42-74.

Sirvent, M.T. (2007). La educación de jóvenes y adultos frente al desafío de los movimientos sociales emergentes en Argentina. Revista Argentina de Sociología, 5(8), 72-91.

Suárez, D. (2011). Indagación pedagógica del mundo escolar y formación docente. La documentación narrativa de experiencias pedagógicas como estrategia de investigación-formación-acción. Revista del IICE, (30), 17-30.

Suárez, D. (2014). Espacio (auto) biográfico, investigación educativa y formación docente en Argentina: un mapa imperfecto de un territorio en expansión. Revista Mexicana de Investigación Educativa, 19(62), 762-786.

Téllez Velasco, D. (2011). Jóvenes nini y profesionistas titi: la estratificación letrada del desempleo. El Cotidiano, 169, 83-96, septiembre-octubre. México.

Terigi, F. (2010). "Las cronologías de aprendizaje: un concepto para pensar las trayectorias escolares". Conferencia: Universidad Nacional de La Pampa. La Pampa, Argentina.

Touraine, A. (1997). ¿Podremos vivir juntos? La discusión pendiente: el destino del hombre en la aldea global. Buenos Aires: Fondo de Cultura Económica.

Fecha de recepción: 2017/11/10; fecha de aceptación: 2018/02/19;

fecha de publicación: 2018/03/01 